\title{
The Electric Conductivity of Dilute Suspensions of Charged Porous Spheres
}

\author{
Yung C. Liu and Huan J. Keh ${ }^{1}$ \\ Department of Chemical Engineering, National Taiwan University, Taipei 106-17, Taiwan, Republic of China
}

Received March 4, 1997; accepted June 2, 1997

The effective electric conductivity of a dilute suspension of polyelectrolyte molecules or charged flocs in an electrolyte solution is analytically studied. The model used for the particles is a porous sphere in which the density of hydrodynamic frictional segments, and therefore also that of the fixed charges, is constant. The equations which govern the electrochemical potential distributions of ionic species and the fluid flow field inside and outside a porous particle migrating in an unbounded solution are linearized assuming that the system is only slightly distorted from equilibrium. $U$ sing a perturbation method, these linearized equations are solved for a porous sphere in a uniform applied electric field with the density of the fixed charges as the small perturbation parameter. An analytical expression for the effective conductivity of a dilute suspension of identical charged porous spheres is obtained from the average electric current density calculated using the solution of electrochemical potential distributions of the ions. The result demonstrates that the presence of the fixed charges in the porous particles can lead to an augmented or a diminished electric conductivity of the suspension relative to that of a corresponding suspension of uncharged porous particles, depending on the characteristics of the electrolyte solution and the suspending particles. When the anionic and cationic diffusion coefficients of a symmetric electrolyte are the same, the correction for the effect of the fixed charges of the particles on the electric conductivity of the suspension is proportional to the square of the fixed charge density. (C) 1997 Academic Press

Key Words: polyelectrolyte particles; porous spheres; electric conductivity; dilute suspensions.

\section{INTRODUCTION}

When an electric field is applied to charged colloidal particles suspended in an electrolyte solution, the particles and the surrounding ions are driven to migrate. As a consequence, the fluid is dragged to flow by the motion of the particles and the ions, and there is an electric current through the suspension to produce. To determine the current density distribution and transport properties such as the electric conductivity, it is necessary to find out not only the local electric

\footnotetext{
${ }^{1}$ To whom correspondence should be addressed.
}

potential but also the local ionic densities and fluid velocity. That is, one must first solve a set of coupled electrokinetic equations to obtain the distributions of electric potential, ionic concentrations and fluid velocity in the electrolyte solution, and then compute the average electric current and conductivity in the suspension.

Theoretical studies on the effective electric conductivity of a suspension of impermeable charged spheres have been made by Saville (1) and O'Brien (2), assuming that the particles and their electric double layers occupy only a small fraction of the total volume. Approximate formulas for the conductivity were obtained using a perturbation method for particles with low $\zeta$ potential immersed in a symmetric electrolyte correct to $O\left(\zeta^{2}\right)$. Their results have some discrepancies with the experimental data reported by Watillon and Stone-Masui (3), who measured the surface conductances of a number of monodisperse polystyrene latices over a range of particle volume fractions. Later, Saville (4) considered the effects of added counterions produced by the particle charging procedure and of nonspecific adsorption which alters the concentrations of ions in the solution outside the double layers, and obtained better agreement between theories and experiments.

The basic equations governing the electric conductivity of a dilute suspension of colloidal particles also describe the electrophoretic phenomena. O'Brien (5) derived the analytical formulas for the electrophoretic mobility and the electric conductivity of a dilute suspension of dielectric spheres with thin but polarized double layers in a general electrolyte solution. Using a similar analysis, O'Brien and Ward (6) also determined the electrophoretic mobility and the effective conductivity of a dilute suspension of randomly oriented spheroids with thin polarized diffuse layers at the particle surfaces. On the other hand, approximate analytical expressions for the electrophoretic mobility and the conductivity of dilute suspensions of colloidal spheres in symmetric electrolytes were obtained by Ohshima et al. (7). These expressions are correct to order $(\kappa a)^{-1}$, where $\kappa$ is the DebyeHuckel parameter (defined by Eq. [34]) and $a$ is the particle radius. When the zeta potential of the particles is small, their reduced result is in agreement with O'Brien's (2). 
In most practical applications, the electric conductivity of a suspension is known from direct measurement and then the zeta potential of dielectric particles in the suspension can be calculated. Similarly, one can also measure the electrophoretic mobility of a particle in order to get the zeta potential. O'Brien and Perrins (8) derived a formula for the electric conductivity of a porous plug composed of closely packed spheres and compared it with the conductivity data for dilute and concentrated dispersions of monodisperse polystyrene particles reported by van der Put and Bijsterbosch (9). They found significant differences between the zeta potentials evaluated from measurements of the electric conductivity and of the electrophoretic mobility. Similar differences were also found by another work (10) in which the conductivities and electrophoretic mobilities of polystyrene latex systems were measured. On the other hand, Stigter (11) developed a theory based on the concepts used to describe the conductivity of strong electrolyte solutions, in which the specific conductance of the suspension was computed by summing the individual contributions of the particle-ion interactions expressed in terms of equivalent conductances. The differences between the kinetic charges calculated from electrophoresis and from conductance in this theory were found to be small and within the errors of the experiments and the theoretical models (12).

A theoretical investigation of the electrokinetic phenomena of charged porous particles was first performed by Hermans and Fujita $(13,14)$. They derived formulas for the electrophoretic mobility of a porous sphere by introducing the Brinkman equation for the internal flow field of the particle and assuming that the electric double layer remains spherically symmetric in the presence of the applied electric field. The effect of the distortion of the counterion atmosphere around a nearly free-drained polyelectrolyte coil under an applied electric field was examined theoretically by Imai and Iwasa (15), and their numerical results agree well with the experimental data (16). Recently, analytical expressions were derived by Liu and Keh (17) for the sedimentation velocity and potential in a dilute suspension of charged porous spheres under the situation that the electric potentials are low. However, the effects of particle charges on the effective conductivity of a suspension of porous particles have not yet been theoretically investigated.

In this work, we study analytically the effective conductivity of a suspension of charged porous particles. The density of charged segments of each porous particle is assumed to be uniform, but no assumption is made as to the thickness of the electric double layer relative to the dimension of the particle. The suspension is sufficiently dilute that the suspended particles occupy only a small fraction of the total volume of the suspension and the double layer surrounding each particle does not overlap with the others. In Section 2, the average electric current density in a dilute suspension of identical charged parti- cles is given as an integral over a large surface enclosing a single particle and its adjacent double layer, and the effective conductivity of the suspension is related to the electrochemical potential energies of the ionic species. In Section 3, we present the fundamental electrokinetic equations and boundary conditions which govern the electrochemical potential distributions, the electrostatic potential profile, and the fluid flow field inside and outside a porous particle migrating in an unbounded solution when a constant electric field is applied. These basic equations are linearized assuming that the ionic concentrations, the electric potential, and therefore the electrochemical potentials have only slight deviations from equilibrium due to the imposed field. The axisymmetric electrophoretic motion of a charged porous sphere in an unbounded electrolyte solution is considered in Section 4. Using the DebyeHuckel approximation, we first get the solution of the equilibrium electric potential distribution. Then the linearized electrokinetic equations are transformed into a set of differential equations by using a regular perturbation method with the density of the fixed charges inside the porous particle as the small perturbation parameter. The perturbed electrochemical potentials of ions and the fluid velocity are determined by solving this set of differential equations subject to the appropriate boundary conditions. An analytical expression for the electric conductivity of a dilute suspension of identical charged porous spheres is obtained. Finally, in Section 5, typical numerical results for the effects of the fixed charges of porous spheres on the effective conductivity of the suspension are presented.

\section{AVERAGE CURRENT DENSITY IN A SUSPENSION OF CHARGED PARTICLES}

We consider a dilute suspension of identical charged particles immersed in a solution containing $M$ ionic species. The particles may be either porous or impermeable to the fluid. It is assumed that the suspension is statistically homogeneous and all effects of its boundaries are ignored. When the isotropic suspension is subjected to a uniform applied electric field $\mathbf{E}_{\infty}$, one has

$$
\mathbf{E}_{\infty}=-\frac{1}{V} \int_{V} \nabla \psi d \mathbf{x},
$$

where $\psi(\mathbf{x})$ is the electric potential at position $\mathbf{x}$ and $V$ denotes a sufficiently large volume of the suspension. There is a resulting volume-averaged current density, which is collinear with $\mathbf{E}_{\infty}$, defined by

$$
\langle\mathbf{i}\rangle=\frac{1}{V} \int_{V} \mathbf{i} d \mathbf{x},
$$


where $\mathbf{i}(\mathbf{x})$ is the current density distribution. The effective electric conductivity $\Lambda$ of the suspension can be assigned by the linear relation

$$
\langle\mathbf{i}\rangle=\Lambda \mathbf{E}_{\infty} .
$$

Since the measured electric field and current density are equal to $\mathbf{E}_{\infty}$ and $\langle\mathbf{i}\rangle$, respectively, Eq. [3] reduces to the usual experimental definition of conductivity, provided that the suspension is everywhere homogeneous.

The current density $\mathbf{i}$ can be written as

$$
\mathbf{i}=\sum_{m=1}^{M} z_{m} e \mathbf{J}_{m}
$$

where $\mathbf{J}_{m}(\mathbf{x})$ and $z_{m}$ are the number flux distribution and the valence of species $m$, respectively, and $e$ is the charge of a proton. If the solution is dilute, the flux $\mathbf{J}_{m}$ is given by

$$
\mathbf{J}_{m}=n_{m} \mathbf{u}-n_{m} \frac{D_{m}}{k T} \nabla \mu_{m}
$$

with the electrochemical potential energy field of the $m$ th species $\mu_{m}(\mathbf{x})$ defined as (7)

$$
\mu_{m}=\mu_{m}^{0}+k T \ln n_{m}+z_{m} e \psi
$$

Here, $\mathbf{u}(\mathbf{x})$ is the fluid velocity field; $n_{m}(\mathbf{x})$ is the concentration distribution of species $m ; D_{m}$ is the diffusion coefficient of species $m$ which is assumed to be constant both inside and outside the particles if they are porous; $k$ is the Boltzmann constant; $T$ is the absolute temperature; and $\mu_{m}^{0}$ is a constant. The first term on the right-hand side of Eq. [5] represents the convection of the ionic species by the fluid and the second term denotes the diffusion and electrically induced migration of the ions.

The linear relation [3] between the volume-average current density and the electric field for a suspension will be exact in the limit as $\mathbf{E}_{\infty} \rightarrow \mathbf{0}$. To calculate the effective conductivity of the suspension we may assume that the intensity of the applied electric field is not high, and hence that the electric double layer surrounding each particle is only slightly distorted from equilibrium by the application of the field. Therefore, the concentration distribution of each ionic species and the electric potential distribution have small deviations from equilibrium, and one can write

$$
\begin{aligned}
n_{m} & =n_{m}^{(\mathrm{eq})}+\delta n_{m}, \\
\psi & =\psi^{(\mathrm{eq})}+\delta \psi, \\
\mu_{m} & =\mu_{m}^{(\mathrm{eq})}+\delta \mu_{m},
\end{aligned}
$$

where $n_{m}^{(\mathrm{eq})}, \psi^{(\mathrm{eq})}$, and $\mu_{m}^{(\mathrm{eq})}$ are the equilibrium distributions of concentration of species $m$, electric potential, and electrochemical potential energy of species $m$, respectively, and $\delta n_{m}, \delta \psi$, and $\delta \mu_{m}$ are the small perturbations to the equilibrium state (in which no external field is applied). The equilibrium concentration of any species is related to the equilibrium potential by the Boltzmann distribution,

$$
n_{m}^{(\mathrm{eq})}=n_{m}^{\infty} \exp \left(-\frac{z_{m} e \psi^{(\mathrm{eq})}}{k T}\right),
$$

where $n_{m}^{\infty}$ is the constant bulk concentration of type- $m$ ions. The perturbation quantity $\delta \mu_{m}$ is linearly related to the others by

$$
\delta \mu_{m}=k T \frac{\delta n_{m}}{n_{m}^{(\mathrm{eq})}}+z_{m} e \delta \psi
$$

Substituting Eqs. [5]- [9] into Eq. [4], using the fact that $\nabla \mu_{m}^{(\mathrm{eq})}=\mathbf{0}$, and neglecting products of the small quantities $\mathbf{u}, \delta n_{m}$, and $\delta \mu_{m}$, one has

$$
\mathbf{i}=\sum_{m=1}^{M} z_{m} e n_{m}^{(\mathrm{eq})}\left(\mathbf{u}-\frac{D_{m}}{k T} \nabla \delta \mu_{m}\right) .
$$

Far from any particle (beyond the double layer), $n_{m}^{(\mathrm{eq})} \rightarrow$ $n_{m}^{\infty}$ and Eq. [10] becomes

$$
\mathbf{i} \rightarrow-\sum_{m=1}^{M} z_{m} e D_{m}\left(\nabla \delta n_{m}+\frac{z_{m} e n_{m}^{\infty}}{k T} \nabla \delta \psi\right)
$$

By adding and subtracting the current density given by the above equation in the integrand of Eq. [2], one obtains

$$
\begin{aligned}
\langle\mathbf{i}\rangle= & -\sum_{m=1}^{M} \frac{z_{m} e D_{m}}{V} \int_{V}\left(\nabla \delta n_{m}+\frac{z_{m} e n_{m}^{\infty}}{k T} \nabla \delta \psi\right) d \mathbf{x} \\
& +\frac{1}{V} \int_{V}\left[\mathbf{i}+\sum_{m=1}^{M} z_{m} e D_{m}\left(\nabla \delta n_{m}+\frac{z_{m} e n_{m}^{\infty}}{k T} \nabla \delta \psi\right)\right] d \mathbf{x} .
\end{aligned}
$$

In a statistically homogeneous suspension, the volume average of $\nabla \delta n_{m}$ is zero. According to the definition of Eq. [1] the first term on the right-hand side of Eq. [12] equals $\Lambda^{\infty} \mathbf{E}_{\infty}$, where

$$
\Lambda^{\infty}=\sum_{m=1}^{M} \frac{z_{m}^{2} e^{2} n_{m}^{\infty} D_{m}}{k T}
$$

which is the electric conductivity of the electrolyte solution 
in the absence of the particles. The integral in the second term on the right-hand side of Eq. [12] can be calculated by first considering for a single particle as if the others were absent and then multiplying the result by the particle number $N$ in the volume $V$, since the integrand vanishes beyond the double layers surrounding the particles and the suspension is assumed to be sufficiently dilute that the double layers do not overlap with one another. Also, the volume integral can be transformed into a surface integral over a spherical boundary of infinite radius containing the single particle at its center. With this arrangement, the second term becomes

$$
\begin{aligned}
\frac{N}{V} \int_{r \rightarrow \infty} & \left(\mathbf{n} \cdot \mathbf{i r}+\sum_{m=1}^{M} \frac{z_{m} e n_{m}^{\infty} D_{m}}{k T} \delta \mu_{m} \mathbf{n}\right) d S \\
& =-\frac{N}{V} \sum_{m=1}^{M} \frac{z_{m} e n_{m}^{\infty} D_{m}}{k T} \int_{r \rightarrow \infty}\left(\mathbf{n} \cdot \nabla \delta \mu_{m} \mathbf{r}-\delta \mu_{m} \mathbf{n}\right) d S,
\end{aligned}
$$

where $\mathbf{r}$ is the position vector relative to the particle center, $r=|\mathbf{r}|$, and $\mathbf{n}$ is the unit vector outwardly normal to the surface of the boundary. To obtain Eq. [14], the requirement of the conservation of electric charge $(\nabla \cdot \mathbf{i}=0)$ and Eq. [11] have been used. Therefore, the average current density given by Eq. [12] can be expressed as

$$
\begin{aligned}
\langle\mathbf{i}\rangle=\Lambda^{\infty} \mathbf{E}_{\infty}-\frac{N}{V} \sum_{m=1}^{M} & \frac{z_{m} e n_{m}^{\infty} D_{m}}{k T} \\
& \times \int_{r \rightarrow \infty}\left(\mathbf{r} \nabla \delta \mu_{m} \cdot \mathbf{n}-\delta \mu_{m} \mathbf{n}\right) d S .
\end{aligned}
$$

The determination of $\delta \mu_{m}$ in the above equation is concerned with the solution of a set of basic electrokinetic equations for the electrolyte around a single particle. These electrokinetic equations for the case of a charged porous particle are described in the next section and their analytical solution for a charged porous sphere with low fixed charge density is presented in Section 4. From this solution we shall derive the effective conductivity of a dilute suspension of identical porous spheres.

\section{BASIC ELECTROKINETIC EQUATIONS FOR A CHARGED POROUS PARTICLE IN AN ELECTRIC FIELD}

In this section we consider a charged porous particle (such as a polyelectrolyte molecule or an aggregate of fine particles bearing surface charges ) of arbitrary shape in an unbounded liquid solution containing $M$ ionic species when a constant electric field $\mathbf{E}_{\infty}$ is applied. In this solvent-permeable and ion-penetrable particle, idealized charged segments are assumed to distribute at a uniform density. Conservation of all species, which do not react with one another, in the steady state requires that

$$
\nabla \cdot \mathbf{J}_{m}=0, \quad m=1,2, \ldots, M
$$

Here, the species fluxes $\mathbf{J}_{m}$ are defined by Eq. [5] taking $\mathbf{u}$ as the fluid velocity relative to the particle.

We assume that the Reynolds number for the fluid motion is very small, so the inertial effect on the fluid momentum balance can be neglected. The fluid flow is governed by the modified Stokes equations,

$$
\begin{aligned}
\eta \nabla^{2} \mathbf{u}-h(\mathbf{x}) f \mathbf{u} & =\nabla p+\sum_{m=1}^{M} z_{m} e n_{m} \nabla \psi, \\
\nabla \cdot \mathbf{u} & =0
\end{aligned}
$$

where $\eta$ is the viscosity of the fluid; $f$ is the friction coefficient inside the porous particle per unit volume of the fluid; $p(\mathbf{x})$ is the dynamic pressure distribution; and $h(\mathbf{x})$ is a unit step function which equals unity if $\mathbf{x}$ is inside the particle, and zero otherwise. In Eq. [17], $\eta$ and $f$ are assumed to be constant. Note that $f$ can be expressed as $6 \pi \eta a_{\mathrm{s}} N_{\mathrm{s}}$, where $N_{\mathrm{s}}$ and $a_{\mathrm{s}}$ are the number density and the Stokes radius, respectively, of the particle segments.

The electric potential $\psi$ and the space charge density are related by Poisson's equation:

$$
\nabla^{2} \psi=-\frac{4 \pi}{\epsilon}\left[\sum_{m=1}^{M} z_{m} e n_{m}+h(\mathbf{x}) Q\right]
$$

Here, $Q$ is the fixed charge density inside the porous particle and $\epsilon=4 \pi \epsilon_{0} \epsilon_{\mathrm{r}}$, where $\epsilon_{\mathrm{r}}$ is the relative permittivity of the electrolyte solution, which is assumed to be the same inside and outside the particle, and $\epsilon_{0}$ is the permittivity of free space. Experimental data for layers of poly (methacrylic acid), poly (4-vinylpyridine) (18), and poly ( $N$-isopropylacrylamide) (19) on the surfaces of polymer particles indicate that the magnitude of $Q$ ranges from very low to as high as $9 \times 10^{6} \mathrm{C} / \mathrm{m}^{3}$, depending on the temperature, $\mathrm{pH}$ value, and ionic strength of the electrolyte solution.

If the strength of the imposed electric field is weak, the deviations in the ionic concentrations and electric potential from equilibrium are so slight that Eqs. [16] and [17] can be linearized. It has been found that the perturbed electrochemical potential energies $\delta \mu_{m}$ and the fluid velocity $\mathbf{u}$ satisfy the following set of electrokinetic equations $(5,7)$ :

$$
n_{m}^{(\mathrm{eq})} \nabla^{2} \delta \mu_{m}+\nabla n_{m}^{(\mathrm{eq})} \cdot \nabla \delta \mu_{m}=\frac{k T}{D_{m}} \nabla n_{m}^{(\mathrm{eq})} \cdot \mathbf{u}
$$




$$
\eta \nabla^{2} \nabla \times \mathbf{u}-h(\mathbf{x}) f \nabla \times \mathbf{u}=\sum_{m=1}^{M} \nabla n_{m}^{(\mathrm{eq})} \times \nabla \delta \mu_{m} .
$$

Substituting Eq. [8] into Eqs. [20] and [21], one has

$$
\nabla^{2} \delta \mu_{m}=\frac{z_{m} e}{k T}\left(\nabla \psi^{(\mathrm{eq})} \cdot \nabla \delta \mu_{m}-\frac{k T}{D_{m}} \nabla \psi^{(\mathrm{eq})} \cdot \mathbf{u}\right)
$$

$$
\nabla^{2} \nabla \times \mathbf{u}-h(\mathbf{x}) \lambda^{2} \nabla \times \mathbf{u}=
$$$$
-\frac{1}{\eta} \sum_{m=1}^{M}\left[\frac{z_{m} e n_{m}^{\infty}}{k T} \exp \left(-\frac{z_{m} e \psi^{(\mathrm{eq})}}{k T}\right) \nabla \psi^{(\mathrm{eq})} \times \nabla \delta \mu_{m}\right]
$$

where $\lambda=(f / \eta)^{1 / 2}$, and the solution of $\psi^{(\mathrm{eq})}$ for the case of a charged porous sphere will be given in the following section. Note that the reciprocal of the parameter $\lambda$ is the shielding length characterizing the extent of flow penetration inside the porous particle. For some model porous particles made of steel wool (in glycerin-water solution) (20) and plastic foam slab (in silicon oil) (21), experimental values of $1 / \lambda$ can be as large as $0.4 \mathrm{~mm}$, while in the charged or uncharged temperature-sensitive poly $(N$ isopropylacrylamide) hydrogel layers on latex particles in electrolyte solutions, values of $1 / \lambda$ were found to be about $1-50 \mathrm{~nm}$ (19).

The conditions inside the particle are

$$
\delta \mu_{m} \text { and } \mathbf{u} \text { are finite. }
$$

The boundary conditions at the surface of the particle $\left(S^{ \pm}\right)$ are

$$
\begin{aligned}
\left.\delta \mu_{m}\right|_{\mathrm{s}^{+}} & =\left.\delta \mu_{m}\right|_{\mathrm{s}^{-}}, \\
\left.\nabla \delta \mu_{m}\right|_{\mathrm{s}^{+}} & =\left.\nabla \delta \mu_{m}\right|_{\mathrm{s}^{-}}, \\
\left.\mathbf{u}\right|_{\mathrm{s}^{+}} & =\left.\mathbf{u}\right|_{\mathrm{s}^{-}}, \\
\left.\mathbf{n} \cdot \boldsymbol{\sigma}\right|_{\mathrm{s}^{+}} & =\left.\mathbf{n} \cdot \boldsymbol{\sigma}\right|_{\mathrm{s}^{-}}
\end{aligned}
$$

Here, $\boldsymbol{\sigma}$ is the hydrodynamic stress of the fluid given by

$$
\boldsymbol{\sigma}=-p \mathbf{I}+\eta\left[\nabla \mathbf{u}+(\nabla \mathbf{u})^{T}\right]
$$

where $\mathbf{I}$ is the unit dyadic. Equations [25a] and [25b] indicate that the concentration and flux of species $m$ and the potential and electric field must be continuous at the particle surface. Equations [25c] and [25d] are the continuity requirement of the fluid velocity and stress tensor.

The conditions far from the particle are

$$
\begin{gathered}
\mathbf{u}=-\mu_{\mathrm{E}} \mathbf{E}_{\infty}, \\
n_{m} \rightarrow n_{m}^{\infty},
\end{gathered}
$$

$$
\psi \rightarrow-\mathbf{E}_{\infty} \cdot \mathbf{x}
$$

Here, $\mu_{\mathrm{E}}$ is the electrophoretic mobility of the charged porous particle, and its expression for a porous sphere will be given in the next section. Because the equilibrium electric potential and concentration of type- $m$ ions in the bulk solution have been set equal to zero and $n_{m}^{\infty}$, respectively, from Eqs. [7], [9], [27b], and [27c] one obtains the following condition for $\delta \mu_{m}$ at large distances from the particle:

$$
\delta \mu_{m} \rightarrow-z_{m} e \mathbf{E}_{\infty} \cdot \mathbf{x}
$$

Equations [17], [18], and [27a] take a reference frame that the porous particle is at rest and the velocity of the fluid at infinity is the particle velocity in the opposite direction.

\section{SOLUTION FOR THE EFFECTIVE CONDUCTIVITY OF A DILUTE SUSPENSION OF CHARGED POROUS SPHERES}

We now consider a charged porous sphere of radius $a$ immersed into an unbounded electrolyte solution under a uniform applied electric field $\mathbf{E}_{\infty}$. The electrophoretic velocity of the particle is $\mu_{\mathrm{E}} E_{\infty} \mathbf{e}_{\mathrm{z}}$, where $\mathbf{e}_{\mathrm{z}}$ is the unit vector in the axial direction and $E_{\infty}=\left|\mathbf{E}_{\infty}\right|$. The origin of the spherical coordinate system $(r, \theta, \phi)$ is taken to be the center of the particle.

We first seek the solution of $\psi^{(\mathrm{eq})}$ which appears in the governing equations [22] and [23] for a charged porous sphere. Substituting the Boltzmann distribution, Eq. [8], into Poisson's equation [19] at equilibrium, one can get the equilibrium Poisson-Boltzmann equation,

$$
\begin{aligned}
\nabla^{2} \psi^{(\mathrm{eq})} & =-\frac{4 \pi}{\epsilon} \\
\times & {\left[\sum_{m=1}^{M} z_{m} e n_{m}^{\infty} \exp \left(-\frac{z_{m} e \psi^{(\mathrm{eq})}}{k T}\right)+h(r) \mathrm{Q}\right], }
\end{aligned}
$$

where $h(r)$ equals unity if $r<a$ and is zero if $r>a$.

The appropriate boundary conditions for the equilibrium potential are

$$
\begin{gathered}
\left.\psi^{(\mathrm{eq})}\right|_{r=0} \text { is finite, } \\
\left.\psi^{\text {(eq) }}\right|_{r=a^{+}}=\left.\psi^{\text {(eq) }}\right|_{r=a^{-}}, \\
\left.\frac{\mathrm{d} \psi^{(\mathrm{eq})}}{\mathrm{d} r}\right|_{r=a^{+}}=\left.\frac{d \psi^{(\mathrm{eq})}}{d r}\right|_{r=a^{-}}, \\
\left.\psi^{(\mathrm{eq})}\right|_{r \rightarrow \infty} \rightarrow 0 .
\end{gathered}
$$


The solution to Eq. [29] satisfying the above boundary conditions is

$$
\psi^{(\mathrm{eq})}=\psi_{\mathrm{eq} 1} \bar{Q}+O\left(\bar{Q}^{2}\right)
$$

Here,

$$
\bar{Q}=\frac{4 \pi e Q}{\epsilon \kappa^{2} k T}
$$

which is the nondimensional charge density of the porous sphere, and (13)

$$
\psi_{\mathrm{eq} 1}(r)=\frac{k T}{e}\left[1-(\kappa a+1) e^{-\kappa a} \frac{\sinh (\kappa r)}{\kappa r}\right], \quad \text { if } r<a
$$

$$
\psi_{\mathrm{eq} 1}(r)=\frac{k T}{e}[\kappa a \cosh (\kappa a)-\sinh (\kappa a)] \frac{e^{-\kappa r}}{\kappa r}, \quad \text { if } r>a,
$$

where $\kappa$ is the reciprocal of the Debye screening length, defined by

$$
\kappa=\left(\frac{4 \pi e^{2}}{\epsilon k T} \sum_{m=1}^{M} z_{m}^{2} n_{m}^{\infty}\right)^{1 / 2}
$$

Note that $\psi^{\text {(eq) }}$ is a function of $r$ only. Expression [31] for $\psi^{(\mathrm{eq})}$ as a power series in the fixed charge density of the porous sphere up to $O(\bar{Q})$ is the equilibrium solution for the linearized Poisson-Boltzmann equation that is valid for small values of the electric potential (the Debye-Huckel approximation). That is, the charge density $Q$ of the porous particle must be small enough for the potential to remain small.

To solve the small quantities $\delta \mu_{m}, \mathbf{u}$, and $\mu_{\mathrm{E}}$ for the case of small parameter $\bar{Q}$, these variables can be written as perturbation expansions in powers of $\bar{Q}$,

$$
\begin{gathered}
\delta \mu_{m}=-z_{m} e E_{\infty} r \cos \theta+\mu_{m 1} \bar{Q}+\mu_{m 2} \bar{Q}^{2}+\cdots \\
\mathbf{u}=\mathbf{u}_{1} \bar{Q}+\mathbf{u}_{2} \bar{Q}^{2}+\cdots \\
\mu_{\mathrm{E}}=\mu_{\mathrm{E} 1} \bar{Q}+\mu_{\mathrm{E} 2} \bar{Q}^{2}+\cdots
\end{gathered}
$$

where the functions $\mu_{m j}, \mathbf{u}_{j}$, and $\mu_{\mathrm{E} j}$, with $j=1,2, \ldots$, are independent of $\bar{Q}$. The zeroth-order terms of $\mathbf{u}$ and $\mu_{\mathrm{E}}$ disappear because an uncharged particle will not move by applying an electric field. Substituting the expansions given by Eq. [35] and $\psi^{\text {(eq) }}$ given by Eq. [31] into the governing equations [22] and [23] and the boundary conditions [24], [25], [27a], and [28], and equating like powers of $\bar{Q}$ on both sides of the respective equations, we obtain a set of differential equations and boundary conditions for each set of the functions $\mu_{m j}, \mathbf{u}_{j}$, and $\mu_{\mathrm{E} j}$ with $j=1,2, \ldots$.

After collecting the $O(\bar{Q})$ terms in the procedure of this regular perturbation, one obtains the following equations:

$$
\nabla^{2} \mu_{m 1}=-\frac{z_{m}^{2} e^{2} E_{\infty}}{k T} \nabla \psi_{\mathrm{eq} 1} \cdot \nabla(r \cos \theta)
$$

$\nabla^{2} \nabla \times \mathbf{u}_{1}-h(r) \lambda^{2} \nabla \times \mathbf{u}_{1}$

$$
=\frac{1}{\eta} \sum_{m=1}^{M} \frac{z_{m}^{2} e^{2} n_{m}^{\infty} E_{\infty}}{k T} \nabla \psi_{\mathrm{eq} 1} \times \nabla(r \cos \theta)
$$

The boundary conditions for $\mu_{m 1}$ and $\mathbf{u}_{1}$ are

$$
\begin{array}{ll}
r=0: & \mu_{m 1} \text { and } \mathbf{u}_{1} \text { are finite; } \\
r=a: & \mu_{m 1} \text { and } \mathbf{u}_{1} \text { are continuous, }
\end{array}
$$

$$
\nabla \mu_{m 1} \text { and } \mathbf{n} \cdot \boldsymbol{\sigma}_{1} \text { are continuous; }
$$

$$
r \rightarrow \infty: \quad \mu_{m 1} \text { are zero, }
$$

$$
\mathbf{u}_{1} \rightarrow-\mu_{\mathrm{E} 1} E_{\infty} \mathbf{e}_{z} \text {. }
$$

The solutions for $\mu_{m 1}$ and the $r$ and $\theta$ components of $\mathbf{u}_{1}$ subject to Eqs. [37] are

$$
\begin{aligned}
\mu_{m 1} & =E_{\infty} F_{m}(r) \cos \theta \\
u_{1 r} & =E_{\infty} F_{u}(r) \cos \theta, \\
u_{1 \theta} & =-E_{\infty} \frac{1}{2 r} \frac{d}{d r}\left[r^{2} F_{u}(r)\right] \sin \theta,
\end{aligned}
$$

with

$$
\begin{array}{r}
F_{m}(r)=z_{m}^{2} e a\left[-\frac{1}{3} \frac{r}{a}+\left(1+\frac{1}{\kappa a}\right) e^{-\kappa a} \frac{\alpha(\kappa r)}{(\kappa r)^{2}}\right], \\
\text { if } r<a, \\
F_{m}(r)=z_{m}^{2} e a\left[-\frac{1}{3}\left(\frac{a}{r}\right)^{2}+\frac{\alpha(\kappa a)}{\kappa a} \frac{\kappa r+1}{(\kappa r)^{2}} e^{-\kappa r}\right], \\
\text { if } r>a
\end{array}
$$

$$
\begin{aligned}
F_{u}(r)= & -\frac{\epsilon k T}{4 \pi \eta e}\left\{\left(\frac{\kappa}{\lambda}\right)^{2}+2(\kappa a+1) e^{-\kappa a} \frac{\kappa^{2}}{\lambda^{2}-\kappa^{2}}\right. \\
& \left.\times\left[\frac{\alpha(\kappa a)}{\alpha(\lambda a)}-\frac{\alpha(\kappa r)}{\alpha(\lambda r)}\right] \frac{\alpha(\lambda r)}{(\kappa r)^{3}}\right\}, \quad \text { if } r<a,
\end{aligned}
$$




$$
\begin{aligned}
F_{u}(r)=-\mu_{\mathrm{E} 1} & +\left[\mu_{\mathrm{E} 1}-\frac{\epsilon k T}{4 \pi \eta e}\left(\frac{\kappa}{\lambda}\right)^{2}\right]\left(\frac{a}{r}\right)^{3} \\
& -\frac{\epsilon k T}{2 \pi \eta e} \alpha(\kappa a)\left[(\kappa r+1) e^{-\kappa r}\right. \\
& \left.-(\kappa a+1) e^{-\kappa a}\right] \frac{1}{(\kappa r)^{3}}, \text { if } r>a,
\end{aligned}
$$

where

$$
\begin{gathered}
\alpha(x)=x \cosh x-\sinh x, \\
\mu_{\mathrm{E} 1}=\frac{\epsilon k T}{4 \pi \eta e}\left\{\left(\frac{\kappa}{\lambda}\right)^{2}+\frac{1}{3}\left(1+e^{-2 \kappa a}-\frac{1-e^{-2 \kappa a}}{\kappa a}\right)\right. \\
+\frac{1}{3}\left(\frac{\kappa^{2}}{\lambda^{2}-\kappa^{2}}\right)\left(1+\frac{1}{\kappa a}\right) \\
\left.\times\left[\left(\frac{\lambda}{\kappa}\right)^{2} \frac{\kappa a\left(1+e^{-2 \kappa a}\right)-1+e^{-2 \kappa a}}{\lambda a \operatorname{coth}(\lambda a)-1}-1+e^{-2 \kappa a}\right]\right\} .
\end{gathered}
$$

Here, the fact that the sum of the hydrodynamic frictional force and electric force exerted on the porous sphere must be zero has been used to determine $\mu_{\mathrm{E} 1}$. Equation [43] for the electrophoretic mobility of a charged porous sphere at low electric potential is the same as that obtained by Hermans and Fujita (13). This formula was also derived by Ohshima (22) who considered the electrophoresis of a soft spherical particle which tends to a charged porous sphere in the absence of the particle core.

Among the $O\left(\bar{Q}^{2}\right)$ terms in the perturbation procedure, the only distribution we need in the following calculations is the electrochemical potential energy $\mu_{m 2}$. If the solution contains only a symmetrically charged, binary electrolyte the equation governing $\mu_{m 2}$ is

$$
\nabla^{2} \mu_{m 2}=\frac{z_{m} e}{k T} \nabla \psi_{\mathrm{eq} 1} \cdot\left(\nabla \mu_{m 1}-\frac{k T}{D_{m}} \mathbf{u}_{1}\right)
$$

The boundary conditions for $\mu_{m 2}$ are also given by Eq. [37] with the subscript 1 of $\mu_{m 1}$ being replaced by 2 . For a general electrolyte there is an extra term on the righthand side of Eq. [44] involving the $O\left(\bar{Q}^{2}\right)$ correction to the equilibrium potential as expressed by Eq. [31]. This extra term considerably complicates the problem. So we consider here only the case of a symmetric electrolyte, in which the $\bar{Q}^{2}$ term in Eq. [31] vanishes and the leading correction to $\psi_{\text {eq } 1} \bar{Q}$ is $O\left(\bar{Q}^{3}\right)$.
The solution for $\mu_{m 2}$ is

$$
\begin{array}{r}
\mu_{m 2}=-\frac{z_{m} e E_{\infty}}{3 k T}\left[\frac{1}{r^{2}} \int_{0}^{r} r^{3}\left(\frac{d F_{m}}{d r}-\frac{k T}{D_{m}} F_{u}\right) \frac{d \psi_{\mathrm{eq} 1}}{d r} d r\right. \\
\left.+r \int_{r}^{\infty}\left(\frac{d F_{m}}{d r}-\frac{k T}{D_{m}} F_{u}\right) \frac{d \psi_{\mathrm{eq} 1}}{d r} d r\right] \cos \theta
\end{array}
$$

where functions $F_{m}(r)$ and $F_{u}(r)$ are given by Eqs. [40] and [41]. Note that $\mu_{m 2}$ takes the same forms both in the regions $r<a$ and $r>a$.

By substituting Eqs. [35a], [38], and [45] into Eq. [15], making relevant calculations, and then comparing the result with Eq. [3], the effective conductivity of a dilute suspension of identical charged porous spheres is obtained as

$$
\begin{aligned}
\Lambda=\Lambda^{\infty}\left\{1-\frac{\varphi}{1-\epsilon_{\mathrm{p}}}\right. & {\left[\Lambda_{1}(\kappa a)^{2} \bar{Q}\right.} \\
& \left.\left.+\Lambda_{2}(\kappa a)^{4} \bar{Q}^{2}+O\left(\bar{Q}^{3}\right)\right]\right\} .
\end{aligned}
$$

Here, $\epsilon_{\mathrm{p}}$ is the porosity of each particle; $\varphi=N(4 / 3) \pi a^{3}(1$ $\left.-\epsilon_{\mathrm{p}}\right) / V$ is the true volume fraction of the porous particles;

$$
\Lambda_{1}=\frac{\sum_{m=1}^{M} z_{m}^{3} n_{m}^{\infty} D_{m}}{\sum_{m=1}^{M} z_{m}^{2} n_{m}^{\infty} D_{m}} \frac{1}{(\kappa a)^{2}}
$$

for a general electrolyte; and

$$
\begin{aligned}
& \Lambda_{1}=\frac{z\left(D_{+}-D_{-}\right)}{D_{+}+D_{-}} \frac{1}{(\kappa a)^{2}}, \\
& \Lambda_{2}=-\frac{\epsilon k^{2} T^{2}}{2 \pi \eta\left(D_{+}+D_{-}\right) e^{2}} A-z^{2} B
\end{aligned}
$$

for a symmetric electrolyte $\left(M=2, z_{+}=-z_{-}=z, n_{+}^{\infty}=\right.$ $n_{-}^{\infty}=n^{\infty}, \Lambda^{\infty}=\left(D_{+}+D_{-}\right) z^{2} e^{2} n^{\infty} / k T$, where subscripts + and - refer to the cation and anion, respectively). In Eq. [48b],

$$
\begin{aligned}
A= & \frac{1}{(\lambda a)^{2}(\kappa a)^{2}}+\frac{2+\kappa a}{(\kappa a)^{8}}[\alpha(\kappa a)]^{2} e^{-2 \kappa a} \\
& -\left(\frac{\kappa^{2}}{\lambda^{2}-\kappa^{2}}\right) \frac{(1+\kappa a)^{2}}{(\kappa a)^{8}} e^{-2 \kappa a}[\alpha(\kappa a) \sinh (\kappa a) \\
& \left.-\sinh ^{2}(\kappa a)+(\kappa a)^{2}\right]+\frac{2 \lambda^{2} \kappa^{2}}{\left(\lambda^{2}-\kappa^{2}\right)^{2}} \frac{(1+\kappa a)^{2}}{(\kappa a)^{8}} \\
& \times \alpha(\kappa a) e^{-2 \kappa a}\left[\left(\frac{\lambda}{\kappa}\right)^{2} \frac{\alpha(\kappa a)}{\alpha(\lambda a)} \sinh (\lambda a)-\sinh (\kappa a)\right],
\end{aligned}
$$




$$
\begin{aligned}
B= & \frac{2(\kappa a)^{2}+3 \kappa a+3}{3(\kappa a)^{7}} \alpha(\kappa a) e^{-\kappa a}-\frac{\kappa a+1}{3(\kappa a)^{5}} e^{-\kappa a} \\
& \times \sinh (\kappa a)-\frac{3}{4} \frac{\kappa a+2}{(\kappa a)^{8}}[\alpha(\kappa a)]^{2} e^{-2 \kappa a} \\
& -\frac{3}{4} \frac{(1+\kappa a)^{2}}{(\kappa a)^{8}} e^{-2 \kappa a}[\alpha(\kappa a) \sinh (\kappa a) \\
& \left.-\sinh ^{2}(\kappa a)+(\kappa a)^{2}\right] .
\end{aligned}
$$

Note that Eq. [46] is correct to order $\varphi,(\kappa a)^{2} \bar{Q}$ and ( $\kappa a)^{4} \bar{Q}^{2}$ are independent of $\kappa$ or $n^{\infty}$, and the result of the $\bar{Q}^{2}$ term is limited to a symmetric electrolyte. As expected, the electric conductivity of a suspension of uncharged porous particles penetrable to electrolyte ions is the same as that in the absence of the particles $\left(\Lambda=\Lambda^{\infty}\right.$ if $Q=0$ ). The coefficient $\Lambda_{1}$ of the $\bar{Q}$ term, which is independent of the shielding parameter $\lambda$, is proportional to $(\kappa a)^{-2}$, and disappears for a symmetric electrolyte when the diffusivities of the cation and anion take the same value. As to the coefficient $\Lambda_{2}$ of the $\bar{Q}^{2}$ term for a symmetric electrolyte, the first term of the right-hand side of Eq. [48b] (which depends on parameters $\kappa a$ and $\lambda a$ ) represents the effect due to the convection of the fluid, while the second term (which is a function of $\kappa a$ only) denotes the effect due to the deviations of the electrochemical potential distributions from their combined equilibrium and applied values. It is understood that the result given by Eqs. [46]- [49] is only valid with the requirements that $\varphi \ll 1$ and $\kappa a\left[\left(1-\epsilon_{\mathrm{p}}\right) / \varphi\right]^{1 / 3} \gg 1$.

When $\kappa a \ll 1$ and $\lambda a$ is moderate, parameters $A$ and $B$ in Eq. [49] have the approximate forms

$$
\begin{aligned}
A & \approx \frac{1}{9}\left[2+\frac{3}{(\lambda a)^{2}}+\frac{2 \sinh (\lambda a)}{\alpha(\lambda a)}\right] \frac{1}{(\kappa a)^{2}}-\frac{1}{3 \kappa a}, \\
B & \approx \frac{1}{36 \kappa a}-\frac{1}{35}
\end{aligned}
$$

When $\kappa a \gg 1$ and $\lambda a$ is still moderate, $A$ and $B$ become

$$
\begin{aligned}
& A \approx \frac{1}{(\lambda a)^{2}(\kappa a)^{2}}+\frac{1}{2(\kappa a)^{5}}, \\
& B \approx \frac{1}{24}\left[\frac{4}{(\kappa a)^{4}}-\frac{9}{(\kappa a)^{5}}\right] .
\end{aligned}
$$

When both $\kappa a$ and $\lambda a$ are large compared with unity, Eq. [49a] reduces to

$$
\begin{aligned}
A \approx & \frac{1}{(\lambda a)^{2}(\kappa a)^{2}}+\frac{1}{4}\left[\frac{1}{(\kappa a)^{5}}-\frac{3}{(\kappa a)^{7}}\right]\left(\frac{\lambda^{2}-2 \kappa^{2}}{\lambda^{2}-\kappa^{2}}\right) \\
& +\frac{\lambda(\kappa a)^{2}-\kappa-2 \lambda}{2 a(\kappa a)^{6}}\left[\frac{\lambda}{\left(\lambda^{2}-\kappa^{2}\right)(\lambda+\kappa)}\right] \cdot
\end{aligned}
$$

The above equation leads to Eq. [51a] when $\kappa a \gg \lambda a$. If $\lambda a \gg \kappa a$, then Eq. [52] is simplified as

$$
\begin{aligned}
A \approx & \frac{1}{4}\left[\frac{1}{(\kappa a)^{5}}-\frac{3}{(\kappa a)^{7}}\right]+\frac{1}{2}\left[\frac{1}{(\kappa a)^{5}}-\frac{2}{(\kappa a)^{7}}\right] \\
& \times\left(\frac{\kappa}{\lambda}\right)+\frac{1}{4}\left[\frac{4}{(\kappa a)^{4}}-\frac{3}{(\kappa a)^{5}}\right]\left(\frac{\kappa}{\lambda}\right)^{2}
\end{aligned}
$$

When $\lambda a \ll 1$ and $\kappa a$ is moderate, parameter $A$ becomes

$$
\begin{aligned}
A \approx & \frac{1}{(\lambda a)^{2}(\kappa a)^{2}}+\frac{2+\kappa a}{(\kappa a)^{8}}[\alpha(\kappa a)]^{2} e^{-2 \kappa a} \\
& +\frac{(1+\kappa a)^{2}}{(\kappa a)^{8}}[\alpha(\kappa a) \sinh (\kappa a) \\
& \left.-\sinh ^{2}(\kappa a)+(\kappa a)^{2}\right] e^{-2 \kappa a} .
\end{aligned}
$$

When both $\lambda a$ and $\kappa a$ are much smaller than unity, $A$ reduces to

$$
A \approx \frac{1}{(\lambda a)^{2}(\kappa a)^{2}}+\frac{1}{15}\left[\frac{4}{(\kappa a)^{2}}-\frac{5}{\kappa a}\right] .
$$

\section{RESULTS AND DISCUSSION}

For an aqueous solution of $\mathrm{KCl}$ in which the cation and anion mobilities are nearly equal, the coefficient $\Lambda_{1}$ of the $\bar{Q}$ term in Eq. [46] is small in magnitude and equal to $-0.017(\kappa a)^{-2}$ (calculated using the values of $\epsilon k^{2} T^{2} / 4 \pi \eta D_{m} z_{m}^{2} e^{2}$ for ions at room temperature listed in Ref. (23)). For other electrolytes in which the ionic mobilities are very different, the magnitude of $\Lambda_{1}$ can be quite large; e.g., $\Lambda_{1}=-0.206(\kappa a)^{-2}$ for $\mathrm{NaCl}$ and $\Lambda_{1}=0.672(\kappa a)^{-2}$ for $\mathrm{HClO}_{4}$. Note that the value of electric conductivity of a suspension of charged porous particles $(\Lambda)$ can be smaller or larger than that of the suspension when the porous particles are uncharged or in the absence of the particles $\left(\Lambda^{\infty}\right)$, depending on the signs of $\Lambda_{1}$ and $Q$. Also, the influence of the presence of the charged particles on the electric conductivity of a suspension is reduced if the concentration of the electrolyte is increased (i.e., $\kappa$ is increased) assuming all other conditions are constant.

The parameters $A$ and $B$ in association with the coefficient 


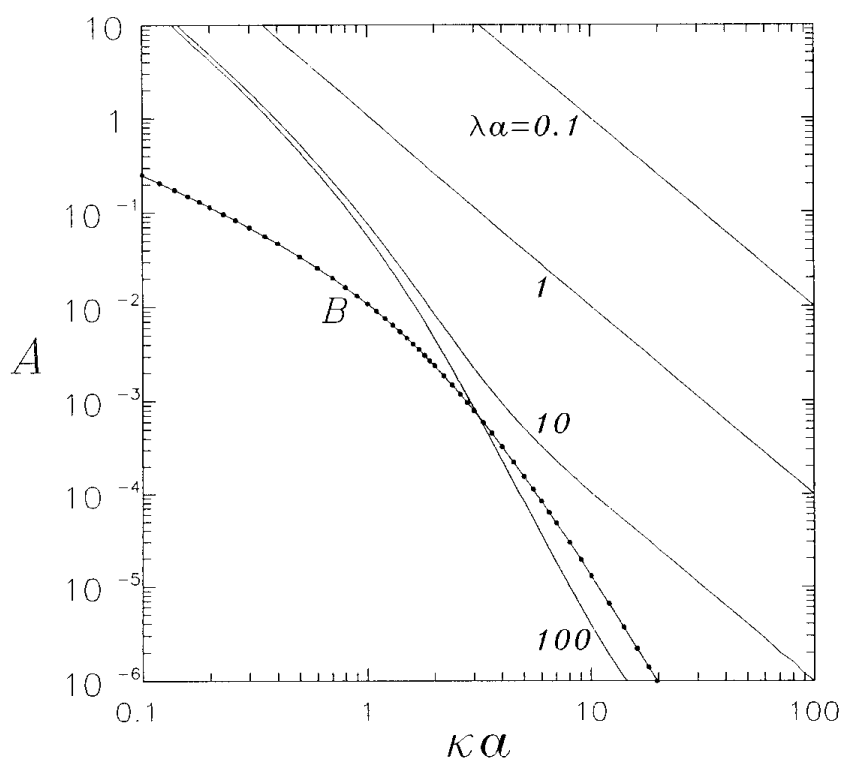

FIG. 1. Plot of the parameter $A$ given by Eq. [49a] for the coefficient $\Lambda_{2}$ versus $\kappa a$ at fixed values of $\lambda a$. The dotted curve is plotted for the parameter $B$ given by Eq. [49b].

$\Lambda_{2}$ by Eq. [48b] can be evaluated for given values of parameters $\kappa a$ and $\lambda a$ using Eq. [49] with the aid of a basic hand calculator, and their results are plotted in Figs. 1 and 2 logarithmically. Figure 1 indicates that both $A$ and $B$ decrease monotonically with increasing $\kappa a$ for a given value of $\lambda a$, and Fig. 2 shows that $A$ is a monotonic decreasing function of $\lambda a$ for a fixed value of $\kappa a$. It can be seen in Fig. 1 that the slope of each curve for $A$ with a constant value of $\lambda a$ equals -2 when $\kappa a \ll 1$ and when $\kappa a \gg 1$, in accordance with Eqs. [50a] and [51a], and that the slope of $B$ equals

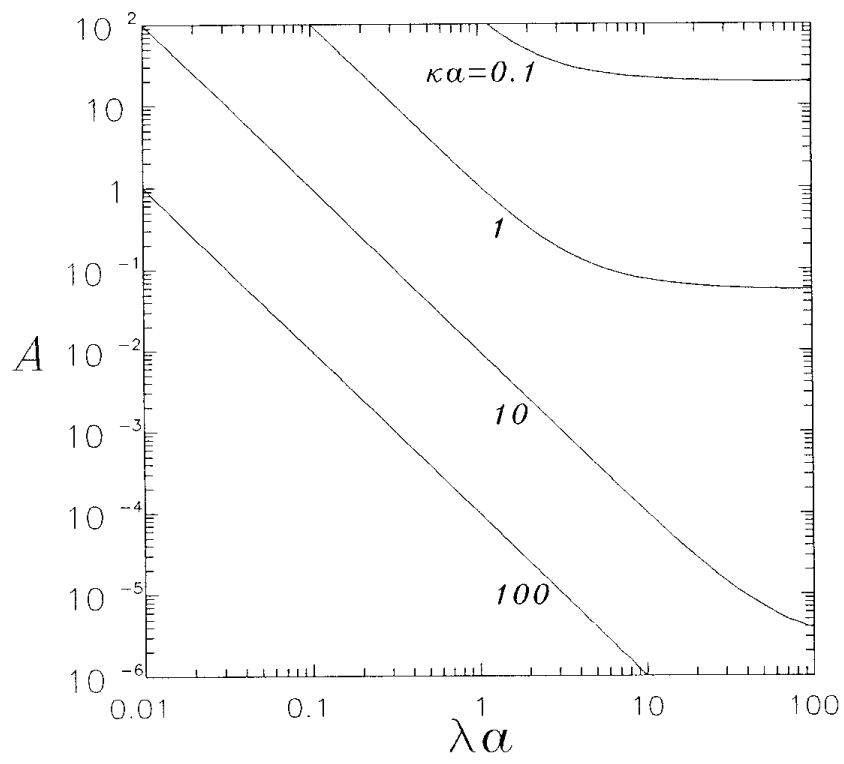

FIG. 2. Plot of the parameter $A$ versus $\lambda a$ at fixed values of $\kappa a$.

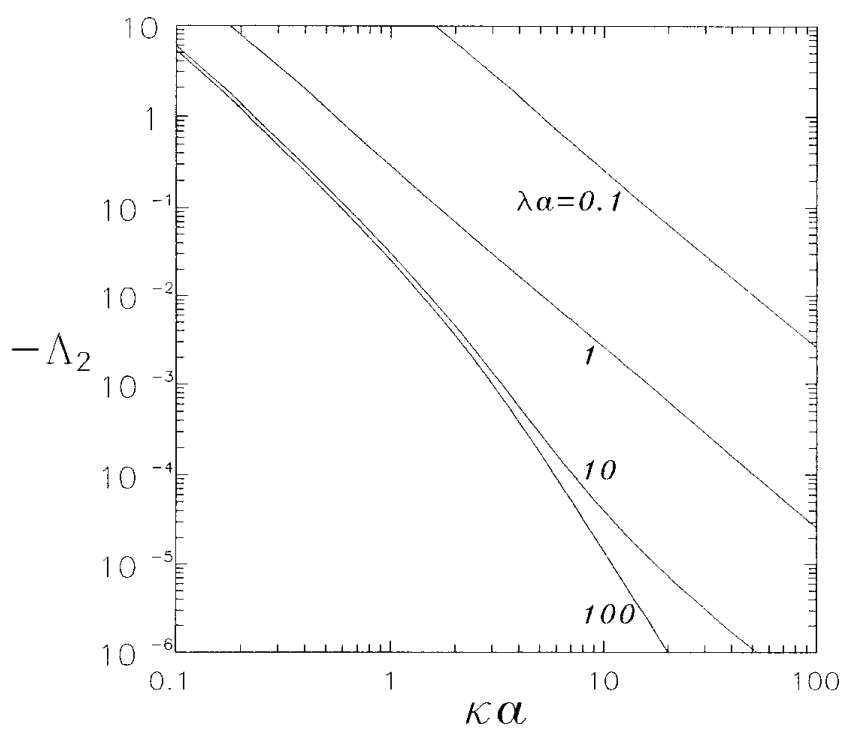

FIG. 3. Plot of the coefficient $\Lambda_{2}$ in Eq. [46] for the effective conductivity of a dilute suspension of identical charged porous spheres in an aqueous solution of $\mathrm{KCl}$ versus $\kappa a$ at fixed values of $\lambda a$.

-1 when $\kappa a \ll 1$ and -4 when $\kappa a \gg 1$, as required by Eqs. [50b] and [51b]. Likewise, Fig. 2 illustrates that the slope of each curve for $A$ with constant $\kappa a$ equals -2 when $\lambda a \ll$ 1 and 0 when $\lambda a \gg 1$, consistent with Eqs. [54] and [53]. Note that the situation of $B>A$ is possible only when $\lambda a$ $\gg 1$ and $\kappa a \geqslant 3$.

Figures 3 and 4 show plots of the coefficient $\Lambda_{2}$ of the $\bar{Q}^{2}$ term in Eq. [46] as a function of parameters $\kappa a$ and $\lambda a$ in an aqueous solution of $\mathrm{KCl}$ where the value of $\epsilon k^{2} T^{2} / 2 \pi \eta\left(D_{+}+D_{-}\right) z^{2} e^{2}=0.2585(23)$ is used in the calculations. As is the case for parameters $A$ and $B$, the

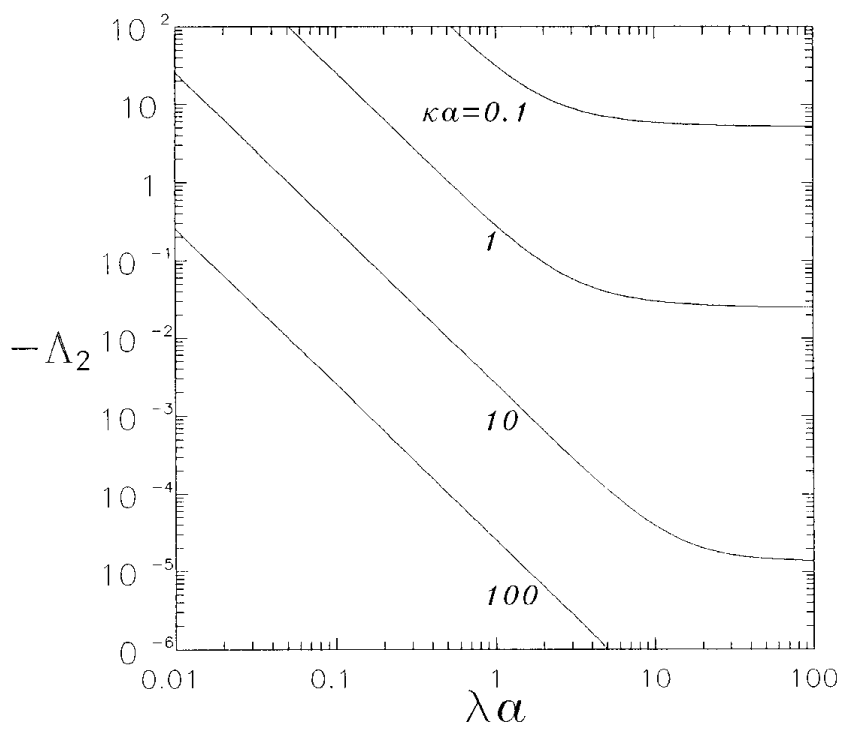

FIG . 4. Plot of the coefficient $\Lambda_{2}$ for a $\mathrm{KCl}$ electrolyte versus $\lambda a$ at fixed values of $\kappa a$. 
magnitude of $\Lambda_{2}$ also decreases monotonically with the increase of $\kappa a$ or $\lambda a$, the other parameter being kept constant. Comparing Figs. 3 and 4 with Figs. 1 and 2, one can find that $\Lambda_{2}$ is dominated by parameter $A$ if $\lambda a<5$ or if $\kappa a<1$, and $\Lambda_{2}$ is dominated by parameter $B$ if $\lambda a$ $\gg 1$ and $\kappa a>3$.

\section{CONCLUDING REMARKS}

The effective electric conductivity of a dilute suspension of charged porous particles in an electrolyte solution is analytically studied in this work. Each porous particle is treated as a solvent-permeable and ion-penetrable object in which fixed-charged groups and frictional segments are distributed at uniform densities. The electric double layer surrounding each particle is assumed not to overlap with the others. The average electric current density passing through the suspension is given by Eq. [15] as an integral over a large spherical surface surrounding a single particle plus its double layer and is related to the electrochemical potential energies of the electrolyte ions. Solving the linearized electrokinetic equations applicable to the system of an isolated porous sphere by a regular perturbation method, we have derived the electrochemical potential distributions of ionic species and the fluid velocity field under the application of a uniform electric field. An analytical expression, Eq. [46], is obtained as a power series in the fixed charge density of the particles up to $O\left(\bar{Q}^{2}\right)$ for the electric conductivity of a dilute suspension of identical charged porous spheres.

It is worth repeating that Eq. [46] with Eqs. [47] and [48] is obtained on the basis of the Debye-Huckel approximation for the equilibrium potential distribution around a porous sphere. A similar formula for the electric conductivity of a dilute suspension of identical impermeable spheres with low $\zeta$ potential was shown to give a good approximation for the case of reasonably high $\zeta$ potential (with an error of about $5 \%$ in a $\mathrm{KCl}$ solution and less than $2 \%$ in a $\mathrm{HClO}_{4}$ solution for the case of $\zeta e / k T=-2$ ) (2). Therefore, our results might be used tentatively for the situation of reasonably high electric potentials. On the other hand, the decay of the density distributions of frictional segments and fixed charges in the porous particle with the distance from the particle center has not been considered in our calculations. In order to see whether our theory can be reasonably extended to the higher values of electric potential or to the nonuniform density distributions of segments and fixed charges, we propose to obtain a numerical solution of the electrokinetic differential equations with no assumption on the magnitude of electric potential allowing the use of arbitrary distributions of charge and fluid drag components in the porous particle and compare it with the approximate solution.

\section{APPENDIX: NOMENCLATURE}

a Radius of a porous sphere (m)

$A, B \quad$ Parameters defined by Eqs. [48b], [49]

$D_{+}, D_{-} \quad$ Diffusion coefficients of cation and anion, respectively $\left(\mathrm{m}^{2} \cdot \mathrm{s}^{-1}\right)$

$D_{m} \quad$ Diffusion coefficient of type- $m$ ions $\left(\mathrm{m}^{2} \cdot \mathrm{s}^{-1}\right)$

$e \quad$ Protonic charge (C)

$\mathbf{E}_{\infty}, E_{\infty} \quad$ Applied electric field $\left(\mathrm{V} \cdot \mathrm{m}^{-1}\right)$

$f$

$F_{m}$

$F_{u}$

$h$

i

$\mathbf{J}_{m}$

$k$

n

$n^{\infty}$

$n_{m}$

$n_{m}^{(\mathrm{eq})}$

$n_{m}^{\infty}$

$N$

\section{Friction coefficient within the porous particle per} unit volume of fluid $\left(\mathrm{kg} \cdot \mathrm{m}^{-3} \cdot \mathrm{s}^{-1}\right)$

Function defined by Eq. [40] $(\mathrm{C} \cdot \mathrm{m})$

Function defined by Eq. [41] $\left(\mathrm{m}^{2} \cdot \mathrm{s}^{-1} \cdot \mathrm{V}^{-1}\right)$

Unit step function

Electric current density $\left(\mathrm{A} \cdot \mathrm{m}^{-2}\right)$

Number flux of type- $m$ ions $\left(\mathrm{s}^{-1} \cdot \mathrm{m}^{-2}\right)$

The Boltzmann constant $\left(1.38 \times 10^{-23} \mathrm{~J} \cdot \mathrm{K}^{-1}\right)$

Unit normal vector outwardly to a surface

Bulk concentration of a symmetric electrolyte $\left(\mathrm{m}^{-3}\right)$

Concentration of type- $m$ ions $\left(\mathrm{m}^{-3}\right)$

Equilibrium concentration of type- $m$ ions $\left(\mathrm{m}^{-3}\right)$

Bulk concentration of type- $m$ ions $\left(\mathrm{m}^{-3}\right)$

Number of the particles in volume $V$ of a suspension

Pressure distribution of the fluid $\left(\mathrm{N} \cdot \mathrm{m}^{-2}\right)$

$p$

$Q \quad$ Fixed charge density inside the porous particle $\left(\mathrm{C} \cdot \mathrm{m}^{-3}\right)$

$=4 \pi e Q / \epsilon \kappa^{2} k T$

Position vector relative to the center of a particle (m)

Radial spherical coordinate $(\mathrm{m})$

Absolute temperature (K)

Fluid velocity $\left(\mathrm{m} \cdot \mathrm{s}^{-1}\right)$

Fluid velocity of the order $\bar{Q}\left(\mathrm{~m} \cdot \mathrm{s}^{-1}\right)$

The $r$ and $\theta$ components of fluid velocity $\mathbf{u}_{1}$ $\left(\mathrm{m} \cdot \mathrm{s}^{-1}\right)$

A sufficiently large volume of the dilute suspension $\left(\mathrm{m}^{3}\right)$

Position vector in the suspension $(\mathrm{m})$

Valence of a symmetric electrolyte

Valence of type- $m$ ions

\section{Greek Letters}

$\alpha \quad$ Function defined by Eq. [42]

$\delta n_{m} \quad$ Perturbed concentration of type- $m$ ions relative to equilibrium $\left(\mathrm{m}^{-3}\right)$

$\delta \mu_{m} \quad$ Perturbed electrochemical potential energy of type- $m$ ions relative to equilibrium $(\mathrm{J})$

$\delta \psi \quad$ Perturbed potential relative to equilibrium (V)

$\epsilon \quad=4 \pi \epsilon_{0} \epsilon_{\mathrm{r}}\left(\mathrm{C}^{2} \cdot \mathrm{J}^{-1} \cdot \mathrm{m}^{-1}\right)$

$\epsilon_{0} \quad$ Permittivity of a vacuum $\left(8.854 \times 10^{-12}\right.$ $\mathrm{C}^{2} \cdot \mathrm{J}^{-1} \cdot \mathrm{m}^{-1}$ ) 
$\epsilon_{\mathrm{p}} \quad$ Porosity of a porous particle

$\epsilon_{\mathrm{r}} \quad$ Dielectric constant of the fluid

$\eta \quad$ Fluid viscosity $\left(\mathrm{kg} \cdot \mathrm{m}^{-1} \cdot \mathrm{s}^{-1}\right)$

$\theta, \phi \quad$ Angular spherical coordinates

$\kappa \quad$ The Debye-Huckel parameter $\left(\mathrm{m}^{-1}\right)$

$\lambda \quad=(f / \eta)^{1 / 2}\left(\mathrm{~m}^{-1}\right)$

$\Lambda$ Electric conductivity of the suspension $\left(\Omega^{-1} \cdot \mathrm{m}^{-1}\right)$

$\Lambda_{1}, \Lambda_{2} \quad$ Coefficients defined by Eqs. [46] - [48]

$\boldsymbol{\sigma}$
$\Lambda^{\infty}$

$\mu_{\mathrm{E}}$

$\mu_{\mathrm{E} 1}$

$\mu_{m}$

$\mu_{m 1}$

$\mu_{m 2}$

$\mu_{m}^{(\mathrm{eq})}$

$\boldsymbol{\sigma}_{1}$

$\varphi$

$\psi$

$\psi^{(\mathrm{eq})}$

$\psi_{\text {eq1 }}$

Electric conductivity of the bulk electrolyte solution $\left(\Omega^{-1} \cdot \mathrm{m}^{-1}\right)$

Electrophoretic mobility of a charged porous particle $\left(\mathrm{m}^{2} \cdot \mathrm{s}^{-1} \cdot \mathrm{V}^{-1}\right)$

Electrophoretic mobility of a charged porous particle of the order $\bar{Q}\left(\mathrm{~m}^{2} \cdot \mathrm{s}^{-1} \cdot \mathrm{V}^{-1}\right)$

Electrochemical potential energy of type- $m$ ions $(\mathrm{J})$

Electrochemical potential energy of type- $m$ ions of the order $\bar{Q}(\mathrm{~J})$

Electrochemical potential energy of type- $m$ ions of the order $\bar{Q}^{2}(\mathrm{~J})$

Equilibrium electrochemical potential energy of type- $m$ ions $(\mathrm{J})$

Hydrodynamic stress $\left(\mathrm{N} \cdot \mathrm{m}^{-2}\right)$

Hydrodynamic stress of the order $\bar{Q}\left(\mathrm{~N} \cdot \mathrm{m}^{-2}\right)$

True volume fraction of the porous particles

Electrical potential of the solution (V)

Equilibrium electrical potential of the solution (V)

Function defined by Eq. [34] (V)

\section{ACKNOWLEDGMENT}

This research was supported by the National Science Council of the Republic of China under Grant NSC86-2214-E-002-013.

\section{REFERENCES}

1. Saville, D. A., J. Colloid Interface Sci. 71, 477 (1979).

2. O'Brien, R. W., J. Colloid Interface Sci. 81, 234 (1981).

3. Watillon, A., and Stone-Masui, J., J. Electroanal. Chem. 37, 143 (1972).

4. Saville, D. A., J. Colloid Interface Sci. 91, 34 (1983).

5. O'Brien, R. W., J. Colloid Interface Sci. 92, 204 (1983).

6. O'Brien, R. W., and Ward, D. N., J. Colloid Interface Sci. 121, 402 (1988).

7. Ohshima, H., Healy, T. W., and White, L. R., J. Chem. Soc., Faraday Trans. 2 79, 1613 (1983).

8. O'Brien, R. W., and Perrins, W. T., J. Colloid Interface Sci. 99, 20 (1984).

9. van der Put, A. G., and Bijsterbosch, B. H., J. Colloid Interface Sci. 75, 512 (1980).

10. Zukoski, C. F., and Saville, D. A., J. Colloid Interface Sci. 107, 322 (1985).

11. Stigter, D., J. Phys. Chem. 83, 1663 (1979).

12. Stigter, D., J. Phys. Chem. 83, 1670 (1979).

13. Hermans, J. J., and Fujita, H., Proc. Akad. Amsterdam B 58, 182 (1955).

14. Hermans, J. J., J. Polymer Sci. 18, 527 (1955).

15. Imai, N., and Iwasa, K., Israel J. Chem. 11, 223 (1973).

16. Noda, I., Nagasawa, M., and Ota, M., J. Amer. Chem. Soc. 86, 5075 (1964).

17. Liu, Y. C., and Keh, H. J., 1996. [Paper presented at Electrokinetic Phenomena '96, Rome ]

18. Aoyanagi, O., Muramatsu, N., Ohshima, H., and Kondo, T., J. Colloid Interface Sci. 162, 222 (1994).

19. Makino, K., Yamamoto, S., Fujimoto, K., Kawaguchi, H., and Ohshima, H., J. Colloid Interface Sci. 166, 251 (1994).

20. Matsumoto, K., and Suganuma, A., Chem. Eng. Sci. 32, 445 (1977).

21. Masliyah, J. H., and Polikar, M., Can. J. Chem. Eng. 58, 299 (1980).

22. Ohshima, H., J. Colloid Interface Sci. 163, 474 (1994).

23. van de Ven, T. G. M., “Colloidal Hydrodynamics,'” p. 86. Academic Press, London, 1989. 